\title{
Increased mortality in Type II diabetic patients using sulphonylurea and metformin in combination: a population-based observational study
}

\author{
J. Olsson ${ }^{1}$, G. Lindberg ${ }^{1}$, M. Gottsäter ${ }^{2}$, K.Lindwall ${ }^{1}$, Å.Sjöstrand ${ }^{3}$, A. Tisell ${ }^{2}$, A. Melander ${ }^{1}$ \\ ${ }^{1}$ The NEPI Foundation, Medical Research Centre, Malmö University Hospital, Malmö, Sweden \\ ${ }^{2}$ Vetlanda Health Care Centre, Vetlanda, Sweden \\ ${ }^{3}$ Tranås Health Care Centre, Tranås, Sweden
}

\section{Abstract}

Aims/hypothesis. This study analysed cause-specific mortality in Type II (non-insulin-dependent) diabetic patients using either sulphonylurea alone or in combination with metformin.

Methods. Patients were followed from the first day they were taking either the combination or sulphonylurea alone. Odds ratios by Cox regression analyses were adjusted for age, sex, duration of diabetes, study area, year of inclusion and fasting blood glucose at inclusion.

Results. We included 169 patients taking sulphonylurea and metformin in combination and 741 patients taking only sulphonylurea. Mean (range) follow-up time was $6.1(0.1-13.0)$ years. The adjusted odds ratio for overall mortality was 1.63 (95\% confidence interval 1.27-2.09) in patients taking sulphonylurea and metformin combination vs those using sulphonylurea alone. For mortality from ischaemic heart disease and stroke the adjusted odds ratios were 1.73 (95\% confi- dence interval 1.17-2.55) and 2.33 (95\% confidence interval 1.17-4.63), respectively.

Conclusion/interpretation. There was a higher cardiovascular mortality in Type II diabetic patients taking sulphonylurea and metformin in combination than in those taking only sulphonylurea. Hence, it cannot be excluded that this kind of combination therapy possibly increases cardiovascular mortality. It is feasible that the increased mortality was secondary to a more aggressive type of diabetes in the patients using sulphonylurea and metformin in combination. Combination therapy is known to promote additional blood glucose reduction but there is as yet no evidence that a sulphonylurea and metformin combination is more beneficial on micro- or macrovascular disease than sulphonylurea or metformin alone. [Diabetologia (2000) 43: 558-560]

Keywords Type II diabetes, pharmacoepidemiology, sulphonylurea, metformin, combination treatment, mortality.
Both sulphonylureas (SU) and metformin (MET) reduce hyperglycaemia in patients with Type II (non-insulin-dependent) diabetes mellitus, but they do so by

Received: 6 December 1999 and in revised form: 7 February 2000

Corresponding author: J. Olsson, MS, The NEPI Foundation, Medical Research Centre, Malmö University Hospital, SE20502 Malmö, Sweden

Abbreviations: SU, Sulphonylurea; MET, metformin; SU + MET, sulphonylurea and metformin in combination; IHD, ischaemic heart disease; $\mathrm{CI}$, confidence interval; ICD, international classification of disease; $\mathrm{HbA}_{1 \mathrm{c}}$, glycated haemoglobin $\mathrm{A}_{1 \mathrm{c}}$. entirely different mechanisms. Therefore, SU and MET can be combined, and a few controlled studies have shown pronounced reductions in hyperglycaemia following treatment with this combination [1-3]. As SU and MET also have beneficial long-term effects on diabetic microvascular disease $[4,5]$ and MET even on macrovascular disease [5], it would seem logical to presume that the combination of SU and MET would be highly beneficial for ischaemic heart disease (IHD) and stroke in patients with Type II diabetes. A UKPDS substudy instead reported a higher risk of diabetes-related death with this type of combination therapy compared with SU therapy alone [5]. Therefore, we decided to analyse cause- 
specific mortality in a Type II diabetic population, including patients either on SU and MET or SU alone.

\section{Subjects and methods}

Subjects. In two Swedish neighbouring towns and municipalities with a population of about 50000,2348 Type II diabetic patients were identified at two primary health care centres and two private practices between 01 January 1984 and 31 December 1994. Type II diabetes was diagnosed according to WHO criteria [6].

Methods. Information was stored under the patient's ten-digit personal identification number such as year of diagnosis, date of medical visit, height and weight, body mass index, fasting blood glucose, glycated haemoglobin $\mathrm{A}_{1 \mathrm{c}}\left(\mathrm{HbA}_{1 \mathrm{c}}\right)$, dosages of oral drugs and insulin, blood pressure, and type of antihypertensive drug when used. The data were derived from medical records and entered into a computerised database. Fasting blood glucose was determined either by Reflolux II or by a routine glucose dehydrogenase method. A correlation test between the two methods was carried out. This gave a correlation of $r=0.98$. All $\mathrm{HbA}_{1 \mathrm{c}}$ determinations were done at the same laboratory using an HPLC-method [7]. Body mass index was calculated as weight $(\mathrm{kg})$ divided by height $(\mathrm{m})^{2}$.

Patients on SU and MET were followed from the first day they were taking the combination. Patients on SU alone (never on MET), were followed from the first time they were using SU according to the patient records. We included 169 patients using SU and MET and 741 patients taking only SU. Due to missing values 86 patients using SU and MET and 272 patients taking SU alone were excluded.

The Swedish mortality register was used to identify who in the cohort died between 01 January 1984 and 31 December 1996. Cause-specific mortality was coded according to the International Classification of Disease (ICD), eighth revision. In case of multiple causes of death, the underlying cause was determined by the Swedish Board of Health and Welfare using a standardised computer algorithm. Ischaemic heart disease (IHD) was defined by ICD-8 codes $410-414$ and stroke by ICD-8 codes 430-438.

All deaths from causes not specified above were referred to as other causes of mortality.

Statistical analysis. Odds ratios calculated by Cox regression analyses were adjusted for age, sex, duration of diabetes, study area, year of inclusion and fasting blood glucose at inclusion. Overall mortality odds ratio was additionally adjusted for body mass index. Statistical significance was assumed at $p$ less than 0.05 .

\section{Results}

At inclusion, patients using SU and MET were 3.6 years younger, had 3.2 years longer diabetes duration and $1.3 \mathrm{mmol} / \mathrm{l}$ higher fasting blood glucose than those using SU alone. Also, body mass index was higher in patients on SU and MET, 28.8 vs 26.3 in patients on only SU. Mean (range) follow-up time was $6.1(0.1-13.0)$ years. Mean follow-up concentrations of fasting blood glucose, $\mathrm{HbA}_{1 \mathrm{c}}$, and blood pressure are given in Table 1. Patients on SU and MET had
Table 1. Mean concentrations of fasting blood glucose, glycated haemoglobin $\mathrm{A}_{1 \mathrm{c}}\left(\mathrm{HbA}_{1 \mathrm{c}}\right)$, and blood pressure during follow-up in users of sulphonylurea as the only oral antihyperglycaemic drug (SU) and in patients using sulphonylurea and metformin in combination $(\mathrm{SU}+\mathrm{MET})$

\begin{tabular}{lcc}
\hline & $\begin{array}{l}\text { SU } \\
(n=741)\end{array}$ & $\begin{array}{l}\text { SU + MET } \\
(n=169)\end{array}$ \\
\hline $\begin{array}{l}\text { Fasting blood glucose (mmol/l), } \\
\text { (no. missing) }\end{array}$ & $8.8(30)$ & $10.3(3)$ \\
$\begin{array}{l}\text { HbA }_{1 \mathrm{c}}(\%), \text { (no. missing) } \\
\begin{array}{l}\text { Systolic blood pressure (mm Hg), } \\
\text { (no. missing) }\end{array}\end{array}$ & $7.3(195)$ & $8.3(30)$ \\
$\begin{array}{l}\text { Diastolic blood pressure (mm Hg), } \\
\text { (no. missing) }\end{array}$ & $82.9(37)$ & $155.4(3)$ \\
\hline
\end{tabular}

(no. missing), number of patients for whom data was missing

Table 2. Odds ratios (OR) and $95 \%$ confidence intervals (95\% CI) associated with use of SU and MET compared with the use of SU, adjusted for age, sex, duration of diabetes, study area, year of inclusion and fasting blood glucose at inclusion

\begin{tabular}{lll}
\hline Cause of mortality & $\begin{array}{l}\text { Multiple causes } \\
\text { OR }(95 \% \mathrm{CI})\end{array}$ & $\begin{array}{l}\text { Underlying cause } \\
\text { OR }(95 \% \mathrm{CI})\end{array}$ \\
\hline IHD & $1.82(1.31-2.53)$ & $1.73(1.17-2.55)$ \\
Stroke & $2.06(1.23-3.45)$ & $2.33(1.17-4.63)$ \\
Other causes & & $1.39(0.95-2.02)$ \\
Non-IHD, non-stroke & $1.17(0.73-1.89)$ & \\
Overall & $1.63(1.27-2.09)$ & $1.63(1.27-2.09)$ \\
\hline
\end{tabular}

$1.5 \mathrm{mmol} / \mathrm{l}$ higher fasting blood glucose and a $1.0 \%$ higher $\mathrm{HbA}_{1 \mathrm{c}}$ than those on SU alone (mean values). Blood pressure differed neither initially nor at follow-up (Table 1).

Compared with SU therapy alone, the odds ratio for overall mortality, adjusted for age, sex, duration of diabetes, study area, year of inclusion and fasting blood glucose at inclusion was 1.63 (95\% CI 1.272.09 ) in those on SU and MET (Table 2). The overall mortality risk estimate remained unchanged when additionally adjusted for body mass index. We excluded 81 patients due to missing data on body mass index.

Adjusted odds ratios for IHD mortality and stroke mortality (underlying cause) were 1.73 (95\% CI 1.17-2.55) and 2.33 (95\% CI 1.17-4.63), respectively. The odds ratios for mortality from causes other than IHD and stroke were not significantly increased (Table 2).

\section{Discussion}

As in the randomised UKPDS substudy [5], our observational study found that cardiovascular mortality, after adjustments for baseline differences, was higher in Type II diabetic patients using SU and MET than in those on SU alone. Due to missing data, only overall mortality risk was adjusted for body mass index at 
inclusion, however, without impact on the risk estimate. Hence, it cannot be ruled out that the combination SU and MET possibly increases cardiovascular mortality compared with the use of SU alone. It seems more likely though that the higher cardiovascular mortality in the SU and MET patients reflected more aggressive diabetes among these patients, as they had a longer duration of diabetes, were younger, were more obese, and had higher glucose concentrations not only at inclusion but even during follow-up.

The higher cardiovascular mortality could also have reflected insufficient effectiveness in the mode of combining SU and MET. It is possibly advantageous to initiate treatment with MET and add SU later, or to combine the two drugs in low doses at the start of the treatment $[1,2]$.

Due to the high quality of the Swedish mortality register, all patients were able to be tracked during follow-up. Thus, the overall mortality estimates are reliable. Knowledge of the underlying cause of death, however, might not be sufficient to identify causal pathways for morbidity and mortality; e.g. stroke could have been caused by a hypoglycaemic event not observed or not registered.

Misclassification of exposure and outcome might have occurred. Patients were classified as using SU and MET or only SU based on notes in their medical records. No information was available on drug treatment before 1984 or for 1995 and 1996, the last two years of follow-up. Some patients possibly discontinued their drugs before the end of follow-up. Misclassification of exposure was most probably random, leading to a potential underestimation of the true risk.

In conclusion, it seems most likely that the higher cardiovascular mortality in patients taking SU and MET was secondary to more aggressive diabetes in patients selected for such therapy. It should not be entirely excluded, however, that the combination possibly enhances cardiovascular mortality. Irrespective of these alternatives it must be emphasised that, while SU and MET are more effective in treating hyperglycaemia than SU or MET alone [1-3], there is no evidence of a corresponding improvement in cardiovascular outcome. Based on the UKPDS data on the combination therapy of metformin and sulphonylurea and based on our study this combination might be harmful.

Acknowledgements. This study was supported by Apoteksbolaget's (The National Corporation of Pharmacies) Fund for Research and Studies in Health Economics and Social Pharmacy, Stockholm, and by the NEPI Foundation (The Network of Pharmacoepidemiology), Malmö and Stockholm, Sweden.

We also want to express our gratitude to the secretaries at the primary health care centres in the towns of Tranås and Vetlanda, and to M. Fahlén, MD, for giving access to a computerised record system.

\section{References}

1. Hermann LS, Scherstén B, Bitzén PO, Kjellström T, Lindegärde F, Melander A (1994) Therapeutic comparison of metformin and sulfonylurea, alone and in various combinations. A double-blind controlled study. Diabetes Care 10: 1100-1109

2. Hermann LS, Schersten B, Melander A (1994) Antihyperglycaemic efficacy, response prediction and dose-response relations of treatment with metformin and sulphonylurea, alone and in primary combination. Diabet Med 10: 953-960

3. DeFronzo RA, Goodman AM (1995) Efficacy of metformin in patients with non-insulin-dependent diabetes mellitus. The multicenter metformin study group. N Engl J Med 333: 541-549

4. Turner RC, Holman RR, Cull CA et al. (1998) Intensive blood-glucose control with sulphonylureas or insulin compared with conventional treatment and risk of complications in patients with type 2 diabetes (UKPDS 33). Lancet 352: 837-853

5. Turner RC, Holman RR, Cull CA et al. (1998) Effect of intensive blood-glucose control with metformin on complications in overweight patients with type 2 diabetes (UKPDS 34). Lancet 352: 854-862

6. World Health Organization Study Group on Diabetes Mellitus (1985) Technical report series No 727, WHO, Geneva

7. Jeppsson JO, Jerntorp P, Sundqvist G, Englund H, Nylund V (1986) Measurement of hemoglobin A1 c by a new liquidchromatographic assay: methodology, clinical utility, and relation to glucose tolerance evaluated. Clin Chem 32: 1867-1872 